\title{
Neumaier graphs with few eigenvalues
}

\author{
Aida Abiad ${ }^{1,2,3} \cdot$ Bart De Bruyn ${ }^{4}$. Jozefien D'haeseleer² . Jack H. Koolen ${ }^{5,6}$
}

Received: 11 July 2020 / Revised: 15 February 2021 / Accepted: 24 February 2021 / Published online: 6 April 2021

(c) The Author(s) 2021

\begin{abstract}
A Neumaier graph is a non-complete edge-regular graph containing a regular clique. In this paper we give some sufficient and necessary conditions for a Neumaier graph to be strongly regular. Further we show that there does not exist Neumaier graphs with exactly four distinct eigenvalues. We also determine the Neumaier graphs with smallest eigenvalue -2 .
\end{abstract}

Keywords Edge-regular graph $\cdot$ Regular clique $\cdot$ Strongly regular graph

Mathematics Subject Classification 05C69 - 05E30

\section{Introduction}

A regular graph is called edge-regular if any two adjacent vertices have the same number of common neighbours. A regular clique in a regular graph is a clique $C$ having the property

This is one of several papers published in Designs, Codes and Cryptography comprising the "Special Issue: The Art of Combinatorics - A Volume in Honour of Aart Blokhuis".

$凶$ Aida Abiad

a.abiad.monge@tue.nl

Bart De Bruyn

Bart.DeBruyn@ugent.be

Jozefien D'haeseleer

Jozefien.Dhaeseleer@ugent.be

Jack H. Koolen

koolen@ustc.edu.cn

1 Department of Mathematics and Computer Science, Eindhoven University of Technology, Eindhoven, The Netherlands

2 Department of Mathematics: Analysis, Logic and Discrete Mathematics, Ghent University, Ghent, Belgium

3 Department of Mathematics and Data Science of Vrije, Universiteit Brussels, Brussels, Belgium

4 Department of Mathematics: Algebra and Geometry, Ghent University, Ghent, Belgium

5 School of Mathematical Sciences, University of Science and Technology of China, Hefei, China

6 CAS Wu Wen-Tsun Key Laboratory of Mathematics, University of Science and Technology of China, Hefei, China 
that every vertex outside $C$ is adjacent to the same positive number of vertices of $C$. In the early 1980s, Neumaier [13] studied regular cliques in edge-regular graphs, and a certain class of designs whose point graphs are strongly regular and contain regular cliques. He then posed the problem of whether there exists a non-complete, edge-regular, non-strongly regular graph containing a regular clique, [13, p. 248].

We define a Neumaier graph as a non-complete edge-regular graph containing a regular clique. A Neumaier graph that is not a strongly regular graph is called a strictly Neumaier graph.

Neumaier graphs have received quite a lot of attention recently. Greaves and Koolen [11] provided the first infinite family of Neumaier graphs, using cyclotomic numbers, showing that strictly Neumaier graphs exist. Goryainov and Shalaginov [10] classified the Cayley-Deza graphs with fewer than 60 vertices, and it turned out that there are four strictly Neumaier graphs with 24 vertices among them. Greaves and Koolen [12] found a new infinite family of strictly Neumaier graphs containing some of the examples found by Goryainov and Shalaginov. Evans, Goryainov and Panasenko [8] obtained some new infinite families of strictly Neumaier graphs by switching in affine polar graphs $\mathrm{VO}^{+}(2 e, 2)$ defined over the binary field. They also showed some general results on Neumaier graphs and their feasible parameter tuples, and presented an application of such results to determine the smallest non-strongly regular Neumaier graph, answering some questions posted by Greaves and Koolen in [11].

We continue the study of Neumaier graphs by studying some of their spectral properties. In particular, in this paper we take a closer look at Neumaier graphs with few distinct eigenvalues. In Sect. 2 we present several combinatorial and spectral conditions under which a Neumaier graph is a strongly regular graph. We also determine the Neumaier graphs with smallest eigenvalue -2 . In Sect. 3 we show some feasibility conditions of Neumaier graphs with four distinct eigenvalues and we use them to prove that there does not exist strictly Neumaier graphs with exactly four distinct eigenvalues.

\section{Strongly regular Neumaier graphs}

In this section we study under which conditions Neumaier graphs have exactly three distinct eigenvalues, that is, we provide several new characterizations of strongly regular Neumaier graphs. A result in this direction was already obtained by Neumaier in Corollary 2.4 of [13]. Recall that not all Neumaier graphs are strongly regular graphs, as it was shown with the constructions by Greaves and Koolen [11,12], and Evans, Goryainov and Panasenko [8].

\subsection{A combinatorial characterization}

Let $\Gamma=(V, E)$ be a non-complete edge-regular graph on $v>0$ vertices having valency $k \geq 1$. We denote by $\lambda$ the constant number of triangles through a given edge. If $\Gamma$ is a complete multipartite graph, then we denote the total number of its multipartite parts by $s+1$. If $\Gamma$ is not a complete multipartite graph, then we show in Lemma 2.2 that $v+\lambda-2 k \neq 0$. In this case, we define $s$ to be the following number:

$$
s:=\frac{-\left(k^{2}-k+\lambda-v \lambda\right)+\sqrt{\left(k^{2}-k+\lambda-v \lambda\right)^{2}+4 k(v-k-1)(v+\lambda-2 k)}}{2(v+\lambda-2 k)} .
$$

In Propositions 2.3 and 2.4 below, we show the following. 
- Every clique $C$ of $\Gamma$ has order at most $s+1$, with equality if and only if $C$ is a regular clique.

- If $\Gamma$ has a regular clique (of order $s+1$ ), then $v \neq s+1$ (as $\Gamma$ is not complete) and every vertex outside $C$ is adjacent to precisely $e:=\frac{(s+1)(k-s)}{v-(s+1)}$ vertices of $C$.

Note that if $\Gamma$ is a Neumaier graph, then there are (regular) cliques of order $s+1$. The main goal of this subsection is to prove the following characterization of a particular family of strongly regular Neumaier graphs.

Theorem 2.1 If $\Gamma$ is a Neumaier graph with the property that every clique of order $e+1$ is contained in a clique of order $s+1$, then $\Gamma$ is strongly regular.

We note that there exist infinite families of Neumaier graphs that have the property mentioned in Theorem 2.1. These include the Neumaier graphs that are complete multipartite graphs and the collinearity graphs of finite generalized quadrangles or finite polar spaces.

We will denote the neighbourhood of a vertex $v$ of $\Gamma$ by $v^{\perp}=\Gamma(v)$. If $v$ is a vertex, then $\Gamma_{i}(v)$ with $i \in \mathbb{N}$ denotes the set of vertices at distance $i$ from $v$. If $v$ and $w$ are two vertices of $\Gamma$, then we write $v \sim w$ or $v \nsim w$ depending on whether $v$ and $w$ are adjacent or not. We denote the edge through two adjacent vertices $v$ and $w$ by $v w$. For any set $X$ of vertices of $\Gamma$, we denote by $\bar{X}:=V \backslash X$ its complement in $V$.

If $\Gamma$ is a complete multipartite graph, then the fact that $k \geq 1$ implies that the number $(s+1)$ of multipartite parts is at least 2 , and the fact that $\Gamma$ is regular and non-complete implies that all multipartite parts have the same order $m \geq 2$. In this case, we have $v=(s+1) m$, $k=s m, \lambda=(s-1) m$ and hence $v+\lambda-2 k=0$. The latter property is sufficient to characterize complete multipartite graphs as the following lemma shows.

Lemma 2.2 We have $v+\lambda-2 k \geq 0$, with equality if and only if $\Gamma$ is a complete multipartite graph.

Proof If $x y$ is an edge of $\Gamma$, then $v \geq\left|x^{\perp} \cup y^{\perp}\right|=2 k-\lambda$, implying that $v+\lambda-2 k \geq 0$. Suppose now that equality holds. Then for every three distinct vertices $x, y$ and $z$ for which $x \sim y$, we have that $z$ is adjacent to at least one of $x, y$. If $u_{1}$ and $u_{2}$ are two non-adjacent vertices and $u_{1} w$ is an edge, then the fact that $u_{1} \nsim u_{2}$ implies that $w \sim u_{2}$. So, the neighbourhoods of two vertices of $\Gamma$ are equal if and only if these vertices are non-adjacent. The non-adjacency relation on the vertex set is thus an equivalence relation and $\Gamma$ is a complete multipartite graph.

Neumaier already observed that if an edge-regular graph has a regular clique, say of order $c$, then any regular clique has order $c$ and any clique with order $c$ is regular [13, Theorem 1.1]. The following is implicitly shown in [13], but we add its proof for completeness.

Proposition 2.3 Suppose $\Gamma$ is not a complete multipartite graph. Every clique $C$ of $\Gamma$ then has order at most $s+1$, and equality occurs if and only if $C$ is a regular clique. In this case, every vertex outside $C$ is adjacent to precisely e vertices of $C$.

Proof Suppose $C$ is a clique of order $\sigma+1 \geq 1$. For every $x \in \bar{C}$, let $e_{x}$ denote the number of vertices in $C$ adjacent to $x$. Then

$$
\sum_{x \in \bar{C}} 1=v-(\sigma+1) .
$$


Counting in two different ways the pairs $(y, x) \in C \times \bar{C}$ with $y \sim x$ gives

$$
\sum_{x \in \bar{C}} e_{x}=(\sigma+1)(k-\sigma) .
$$

Counting in two different ways the pairs $\left(y_{1}, y_{2}, x\right) \in C \times C \times \bar{C}$ with $y_{1} \neq y_{2}$ and $y_{1} \sim x \sim y_{2}$ gives

$$
\sum_{x \in \bar{C}} e_{x}\left(e_{x}-1\right)=(\sigma+1) \sigma(\lambda-(\sigma-1)) .
$$

From (2) and (3), we find

$$
\sum_{x \in \bar{C}} e_{x}^{2}=(\sigma+1)\left(\sigma \lambda-\sigma^{2}+k\right) .
$$

From the Cauchy-Schwartz inequality, we know that

$$
\left(\sum e_{x}\right)^{2} \leq\left(\sum 1\right) \cdot\left(\sum e_{x}^{2}\right)
$$

with equality if and only if all $e_{x}$ 's are equal, i.e., if and only if $\{C, \bar{C}\}$ is an equitable partition. We thus find

$$
\begin{aligned}
& (\sigma+1)^{2}(k-\sigma)^{2} \leq(v-(\sigma+1))(\sigma+1)\left(k+\sigma \lambda-\sigma^{2}\right), \\
& (\sigma+1)\left(k^{2}-2 \sigma k+\sigma^{2}\right) \leq(v-\sigma-1)\left(k+\sigma \lambda-\sigma^{2}\right), \\
& k^{2} \sigma-2 \sigma^{2} k+\sigma^{3}+k^{2}-2 \sigma k+\sigma^{2} \leq v k+v \lambda \sigma-v \sigma^{2}-\sigma k-\sigma^{2} \lambda+\sigma^{3}-k-\sigma \lambda+\sigma^{2},
\end{aligned}
$$

i.e.,

$$
(v+\lambda-2 k) \sigma^{2}+\left(k^{2}-k+\lambda-v \lambda\right) \sigma+\left(k^{2}+k-v k\right) \leq 0 .
$$

By Lemma 2.2, we know that $v+\lambda-2 k>0$. As $k \geq 1$ and $\Gamma$ is not complete, we also have $k^{2}+k-v k=-k(v-(k+1))<0$. So, the quadratic equation (5) in the variable $\sigma$ has two real roots $s_{1}$ and $s_{2}$ with $s_{1}<0$ and $s_{2}>0$. A straightforward computation shows that $s_{2}=s$. So, (5) implies that $\sigma \leq s$. By the above we also know that $\sigma=s$ if and only if all $e_{x}$ 's are equal, i.e., if and only if $C$ is a regular clique. If all $e_{x}$ 's are equal, then they are equal to their average value $\left(\sum e_{x}\right) \cdot\left(\sum 1\right)^{-1}=\frac{(\sigma+1)(k-\sigma)}{v-\sigma-1}=\frac{(s+1)(k-s)}{v-s-1}=e$.

A similar property as in Proposition 2.3 holds in case $\Gamma$ is a complete multipartite graph. Note that if $\Gamma$ is a complete multipartite graph having $s+1 \geq 2$ parts of size $m \geq 2$, then $e=\frac{(s+1)(k-s)}{v-(s+1)}=\frac{(s+1)(m s-s)}{(s+1) m-(s+1)}=s$.

Proposition 2.4 Suppose $\Gamma$ is a complete multipartite graph. Every clique $C$ of $\Gamma$ then has order at most $s+1$, and equality occurs if and only if $C$ is a regular clique. In this case, every vertex outside $C$ is adjacent to precisely $e=s$ vertices of $C$.

Proof Recall that $\Gamma$ has exactly $s+1$ multipartite parts. The obvious bound for $|C|$ is therefore $s+1$. If $|C|=s+1$, then $C$ contains exactly one point of each of the $s+1$ multipartite parts. In this case, every point outside $C$ is adjacent to exactly $s$ vertices of $C$. If $0<|C|<s+1$, then there is a point outside $C$ that is adjacent to all $|C|$ vertices of $C$ and a point outside $C$ that is adjacent to exactly $|C|-1$ vertices of $C$, proving that $C$ cannot be a regular clique. 
Our next aim is to prove Theorem 2.1. So, from now on, we assume that $\Gamma$ is a Neumaier graph with the property that every clique of order $e+1$ is contained in a clique of order $s+1$. Our intention is thus to prove that $\Gamma$ is strongly regular. In the sequel, a clique of order $l$ will shortly be called an l-clique. By Propositions 2.3 and 2.4, we have:

Lemma 2.5 Every $(s+1)$-clique is a maximum clique. Hence, $e<s+1$.

Lemma 2.6 Every $(e+1)$-clique $H$ is contained in a unique $(s+1)$-clique.

Proof This immediately follows from Lemma 1.5(i) in [13]. If $C_{1}$ and $C_{2}$ were two distinct $(s+1)$-cliques containing $H$, then every vertex of $C_{2} \backslash C_{1}$ is adjacent to all $\left|C_{1} \cap C_{2}\right| \geq e+1$ vertices of $C_{1} \cap C_{2}$, an obvious contradiction.

Lemma 2.7 Every clique $H$ is contained in an $(s+1)$-clique.

Proof Let $C^{\prime}$ denote an $(s+1)$-clique for which $\left|H \cap C^{\prime}\right|$ is maximal. We show that $H \subseteq C^{\prime}$. If this were not the case, then there exists a vertex $x \in H \backslash C^{\prime}$. The set $x^{\perp} \cap C^{\prime}$ then contains $H \cap C^{\prime}$ and so the unique $(s+1)$-clique $C^{\prime \prime}$ containing the $(e+1)$-clique $\{x\} \cup\left(x^{\perp} \cap C^{\prime}\right)$ would have a larger intersection with $H$ than $C^{\prime}$.

Lemma 2.8 The graph $\Gamma$ has diameter 2.

Proof Since $\Gamma$ is not a complete graph, it suffices to prove that any two distinct non-adjacent vertices $x$ and $y$ have distance 2. By Lemma 2.7, there exists an $(s+1)$-clique $C$ through $x$. Then $y \notin C$ and $y$ is adjacent to $e \geq 1$ vertices of $C$. So, $x$ and $y$ lie at distance 2 from each other.

Lemma 2.9 If $E_{1}$ and $E_{2}$ are two edges for which $\left|E_{1} \cap E_{2}\right|=1$ and $E_{1} \cup E_{2}$ is not a clique, then they are contained in the same number of $(s+1)$-cliques.

Proof Put $E_{1}=x y_{1}, E_{2}=x y_{2}$ and let $i \in\{1,2\}$. For every $(s+1)$-clique $C_{i}$ through $E_{i}$, there exists a unique $(s+1)$-clique $C_{3-i}$ through $E_{3-i}$ intersecting $C_{i}$ in exactly $e$ vertices. Indeed, this clique necessarily coincides with the unique $(s+1)$-clique containing the $(e+1)$-clique $\left\{y_{3-i}\right\} \cup\left(y_{3-i}^{\perp} \cap C_{i}\right)$. The lemma then follows by counting in two ways the pairs $\left(C_{1}, C_{2}\right)$, where each $C_{i}, i \in\{1,2\}$, is an $(s+1)$-clique through $E_{i}$ and $\left|C_{1} \cap C_{2}\right|=e$.

Lemma 2.10 If $E_{1}$ and $E_{2}$ are two edges for which $\left|E_{1} \cap E_{2}\right|=1$ and $E_{1} \cup E_{2}$ is a clique, then they are contained in the same number of $(s+1)$-cliques.

Proof For any two adjacent vertices $u$ and $v$, let $N_{u v}$ denote the number of $s+1$-cliques containing $\{u, v\}$. Put $E_{1}=x y_{1}$ and $E_{2}=x y_{2}$. Let $\eta$ denote the number of vertices adjacent to $x, y_{1}$ and $y_{2}$. Then for every $i \in\{1,2\}$, the set $A_{i}$ of vertices distinct from $y_{3-i}$ adjacent to $x$ and $y_{i}$ but not to $y_{3-i}$ has order $\lambda-\eta-1$.

Every $(s+1)$-clique through $E_{i}$ not containing $y_{3-i}$ contains $s+1-e>0$ elements of $A_{i}$, namely the $s+1-e$ vertices of $S_{i} \backslash y_{3-i}^{\perp}$. So, if $\lambda-\eta-1=0$, then both $N_{x y_{1}}$ and $N_{x y_{2}}$ are equal to the number of $(s+1)$-cliques through $\left\{x, y_{1}, y_{2}\right\}$. In the sequel, we may therefore assume that $\left|A_{1}\right|=\left|A_{2}\right|=\lambda-\eta-1>0$.

For every $z_{1} \in A_{1}$, we show that the number of (s+1)-cliques containing $\left\{x, y_{1}, z_{1}\right\}$ equals the number of $(s+1)$-cliques containing $\left\{x, y_{1}, y_{2}\right\}$. Put $T_{1}=\left\{x, y_{1}, z_{1}\right\}, T_{2}=\left\{x, y_{1}, y_{2}\right\}$, $u_{1}=z_{1}$ and $u_{2}=y_{2}$. Note that $u_{1} \nsim u_{2}$. For every $i \in\{1,2\}$ and every clique $C_{i}$ of order $s+1$ through $T_{i}$, there then exists a unique clique $C_{3-i}$ of order $s+1$ through $T_{3-i}$ intersecting 
$C_{i}$ in a set of order $e$. This clique $C_{3-i}$ is precisely the unique $(s+1)$-clique containing the $(e+1)$-clique $u_{3-i} \cup\left(u_{3-i}^{\perp} \cap C_{i}\right)$. The claim that the numbers of $(s+1)$-cliques coincide then follows from counting in two ways all pairs $\left(C_{1}, C_{2}\right)$ satisfying $T_{1} \subseteq C_{1}, T_{2} \subseteq C_{2}$ and $\left|C_{1} \cap C_{2}\right|=e$.

In a completely similar way, one proves that if $z_{2} \in A_{2}$, then the number of $(s+1)$-cliques containing $\left\{x, y_{2}, z_{2}\right\}$ equals the number of $(s+1)$-cliques containing $\left\{x, y_{1}, y_{2}\right\}$. If $z_{1} \in A_{1}$ and $z_{2} \in A_{2}$, we thus see that the number of $(s+1)$-cliques containing $\left\{x, y_{1}, z_{1}\right\}$ coincides with the number of $(s+1)$-cliques containing $\left\{x, y_{2}, z_{2}\right\}$. We call this number $\Omega$.

We count the number of $(s+1)$-cliques containing $E_{1}$, but not $E_{2}$. If $C$ is such a clique, then $C$ contains $s+1-e>0$ vertices of $A_{1}$. As every vertex of $A_{1}$ is contained in $\Omega$ $(s+1)$-cliques together with $E_{1}$, and no such $(s+1)$-clique contains $E_{2}$, we see that the number of $(s+1)$-cliques containing $E_{1}$ but not $E_{2}$ is equal to

$$
\frac{\left|A_{1}\right| \cdot \Omega}{s+1-e}=\frac{(\lambda-\eta-1) \Omega}{s+1-e} .
$$

In a similar way one proves that the total number of $(s+1)$-cliques containing $E_{2}$ but not $E_{1}$ equals $\frac{(\lambda-\eta-1) \Omega}{s+1-e}$. To find the total number of $(s+1)$-cliques through $E_{i}, i \in\{1,2\}$, we should still add to this number the total number of $(s+1)$-cliques containing $\left\{x, y_{1}, y_{2}\right\}$. But this extra contribution is constant for both $E_{1}$ and $E_{2}$.

By Lemmas 2.9, 2.10 and the fact that $\Gamma$ is connected, we have:

Corollary 2.11 The number of $(s+1)$-cliques through an edge is constant.

As $\Gamma$ is regular of valency $k \geq 1$, this immediately implies the following via a double counting.

Corollary 2.12 The number of $(s+1)$-cliques through a vertex is constant.

By Lemma 2.8, Corollaries 2.11, 2.12 and Proposition A2 of [6], we obtain the desired result which proves Theorem 2.1:

Corollary 2.13 The graph $\Gamma$ is strongly regular.

\subsection{Characterizations of strongly regular graphs using eigenvalues}

In this section we present some new eigenvalue conditions under which a connected and regular graph is strongly regular. We thus relax the conditions of requiring edge-regularity and a regular clique (i.e., a Neumaier graph) from Sect. 2.1.

First we need to introduce some definitions. Let $\Gamma=(V, E)$ be a graph on $v \geq 3$ vertices that is not a complete multipartite graph, in particular, $\Gamma$ is not a complete graph nor a graph without edges.

We denote by $\bar{k}=\frac{2 \cdot|E|}{v}>0$ the average degree of the vertices of $\Gamma$. We put $\bar{\lambda}:=\frac{6 N}{v \bar{k}}$, where $N$ is the total number of triangles of $\Gamma$. If we define $\lambda_{x y}:=\left|\Gamma_{1}(x) \cap \Gamma_{1}(y)\right|$ for every edge $x y$ of $\Gamma$, then as $\sum_{x y \in E} \lambda_{x y}=3 N$ and $|E|=\frac{v \bar{k}}{2}$, we see that $\bar{\lambda}$ is the average of the $\lambda_{x y}$ 's with $x y \in E$.

In Lemmas 2.17 and 2.18 below, we show that $v>\bar{k}+1$ and $v+\bar{\lambda}-2 \bar{k}>0$. This implies that the quadratic polynomial

$$
(v+\bar{\lambda}-2 \bar{k}) X^{2}+\left(\bar{k}^{2}-\bar{k}+\bar{\lambda}-\bar{\lambda} v\right) X-\bar{k}(v-\bar{k}-1) \in \mathbb{R}[X]
$$


has two real roots, a negative one and a positive one which we denote by $\bar{s}$. We thus have

$$
\bar{s}=\frac{-\left(\bar{k}^{2}-\bar{k}+\bar{\lambda}-\bar{\lambda} v\right)+\sqrt{\left(\bar{k}^{2}-\bar{k}+\bar{\lambda}-\bar{\lambda} v\right)^{2}+4 \bar{k}(v-\bar{k}-1)(v+\bar{\lambda}-2 \bar{k})}}{2(v+\bar{\lambda}-2 \bar{k})}>0 .
$$

We also define

$$
\bar{\mu}:=\frac{\bar{k}(\bar{k}-\bar{\lambda}-1)}{v-\bar{k}-1}, \quad \theta_{m}:=-\frac{\bar{k}}{\bar{s}}, \quad \theta_{M}:=\frac{\bar{k}-\bar{\mu}}{\bar{k}} \bar{s} .
$$

Note that $\bar{\mu}$ is not $\mu$ average. If one replaces $\bar{k}^{2}$ by $\overline{k^{2}}$, then one obtains $\mu$ average, so by Cauchy the latter is at least the former. If $\Gamma$ is a strongly regular graph with parameters $(v, k, \lambda, \mu)$, then $(\bar{k}, \bar{\lambda}, \bar{\mu})=(k, \lambda, \mu)$ and we will see that $k, \theta_{M}$ and $\theta_{m}$ are the eigenvalues of $\Gamma$ (see Lemma 2.21 and its ensuing remark). If $\Gamma$ is a general graph, then we can interpret $\theta_{M}$ and $\theta_{m}$ by their algebraic definitions, depending on $\bar{k}, \bar{s}$ and $\bar{\mu}$.

Our main result in this section is as follows.

Theorem 2.14 Suppose $\Gamma$ is a connected regular graph that is not a complete multipartite graph. If $\theta_{\operatorname{Max} 2}$ is the second largest eigenvalue and $\theta_{\min }$ the smallest eigenvalue of $\Gamma$, then

(a) $\theta_{\min } \leq \theta_{m}$ with equality if and only if $\Gamma$ is strongly regular, and

(b) $\theta_{\text {Max } 2} \geq \theta_{M}$, and equality holds if and only if $\Gamma$ is strongly regular.

We observe that the above result resembles a bit the first lemma in the work by Van Dam and Haemers on spectral characterization of distance regular graphs with diameter 3 [15]. In [15] the authors characterize strongly regular graphs, as we do in the above result, by using eigenvalues of the quotient matrix of local distance partition. In this regard, our Theorem 2.14 looks more global.

In what follows we prove Theorem 2.14 as a special case of a more general result on arbitrary (not necessarily regular) graphs. First we recall some known facts about the spectrum of a graph.

Lemma 2.15 (a) If $\Gamma^{\prime}$ is a graph having precisely one eigenvalue, then this eigenvalue is 0 and $\Gamma^{\prime}$ has no edges.

(b) If $\Gamma^{\prime}$ is a graph having precisely two eigenvalues, then the connected components of $\Gamma^{\prime}$ are complete graphs of the same order $t \geq 2$.

(c) Every connected regular graph having precisely three eigenvalues is strongly regular.

We denote by $\Omega$ the multiset of order $v$ whose elements are the eigenvalues of $\Gamma$ (taking into account their multiplicities). For every $\theta \in \Omega$, we denote by $\Omega_{\theta}$ the multiset of order $v-1$ obtained from $\Omega$ by removing one copy of $\theta$.

Lemma 2.16 We have

$$
\sum_{\theta \in \Omega} \theta=0, \quad \sum_{\theta \in \Omega} \theta^{2}=v \bar{k}, \quad \sum_{\theta \in \Omega} \theta^{3}=v \bar{k} \bar{\lambda} .
$$

Proof Put $S_{1}:=\sum_{\theta \in \Omega} \theta, S_{2}:=\sum_{2} \theta \theta^{\prime}$ and $S_{3}:=\sum_{3} \theta \theta^{\prime} \theta^{\prime \prime}$, with $\Sigma_{2}$ the summation over all $\left\{\theta, \theta^{\prime}\right\} \in\left(\begin{array}{c}\Omega \\ 2\end{array}\right)$ and $\Sigma_{3}$ the summation over all $\left\{\theta, \theta^{\prime}, \theta^{\prime \prime}\right\} \in\left(\begin{array}{c}\Omega \\ 3\end{array}\right)$. If $x^{n}+c_{1} x^{n-1}+c_{2} x^{n-2}+$ $\cdots+c_{n}=\prod_{\theta \in \Omega}(x-\theta)$ is the characteristic polynomial of $\Gamma$, then $S_{1}=-c_{1}=0, S_{2}=c_{2}$ and $S_{3}=-c_{3}$. As the number of edges is $\frac{v \bar{k}}{2}$ and the number of triangles is $\frac{v \bar{k} \bar{\lambda}}{6}$, we have $c_{2}=-\frac{v \bar{k}}{2}$ and $c_{3}=-\frac{v \bar{k} \bar{\lambda}}{3}$. So, $S_{2}=-\frac{v \bar{k}}{2}, S_{3}=\frac{v \bar{k} \bar{\lambda}}{3}$ and $\sum_{\theta \in \Omega} \theta^{2}=S_{1}^{2}-2 S_{2}=v \bar{k}$ and $\sum_{\theta \in \Omega} \theta^{3}=S_{1}^{3}-3 S_{1} S_{2}+3 S_{3}=v \bar{k} \bar{\lambda}$. 
Alternatively, the previous Lemma 2.16 follows easier from the traces of powers of $A$. The following lemma is a straight-forward result.

Lemma 2.17 We have $v>\bar{k}+1$.

Proof For every vertex $x$ of $\Gamma$, we have $1+\left|\Gamma_{1}(x)\right|=\left|\{x\} \cup \Gamma_{1}(x)\right| \leq|V|=v$. Summing over all vertices $x$ of $\Gamma$, we find $v+v \cdot \bar{k} \leq v^{2}$, i.e. $v \geq \bar{k}+1$. If equality occurs, then $\{x\} \cup \Gamma_{1}(x)=V$ for every vertex $x$. This is only possible when $\Gamma$ is a complete graph, contrary to our assumption. So, $v>\bar{k}+1$.

The following lemma concerns the complementary $\mu$ somehow averaged.

Lemma 2.18 We have $v+\bar{\lambda}-2 \bar{k}>0$.

Proof Put $k_{x}:=\left|\Gamma_{1}(x)\right|$ for every vertex $x$ of $\Gamma$. For every edge $x y$ of $\Gamma$, we have $v=|V| \geq$ $\left|\Gamma_{1}(x) \cup \Gamma_{1}(y)\right|=k_{x}+k_{y}-\lambda_{x y}$. Summing over all edges $x y$ of $\Gamma$, we find $\sum_{x \in V} k_{x}^{2}-|E| \cdot \bar{\lambda} \leq$ $v \cdot|E|$, i.e.

$$
\frac{2}{v \bar{k}} \sum_{x \in V} k_{x}^{2}-\bar{\lambda} \leq v
$$

Now, by the Cauchy-Schwartz inequality, we have

$$
v^{2} \bar{k}^{2}=\left(\sum_{x \in V} k_{x}\right)^{2} \leq\left(\sum_{x \in V} 1\right) \cdot\left(\sum_{x \in V} k_{x}^{2}\right)=v \cdot \sum_{x \in V} k_{x}^{2},
$$

i.e.

$$
v \bar{k}^{2} \leq \sum_{x \in V} k_{x}^{2}
$$

By (6) and (8), we thus have $2 \bar{k}-\bar{\lambda} \leq v$, i.e. $v+\bar{\lambda}-2 \bar{k} \geq 0$. In case of equality, so if $v+\bar{\lambda}-2 \bar{k}=0$, we know by the above that

(a) $\Gamma_{1}(x) \cup \Gamma_{1}(y)=V$ for every edge $x y$ of $\Gamma$;

(b) all $k_{x}$ 's are equal, necessarily to $k:=\bar{k}$ (as equality holds in (7)).

Condition (a) implies that $k_{x}+k_{y}-\lambda_{x y}=v$ for every edge $x y$. As $k_{x}=k_{y}=k$ and $v+\bar{\lambda}-2 k=0$, we thus have that $\lambda_{x y}=\bar{\lambda}$ for every edge $x y$ of $\Gamma$. The graph $\Gamma$ is thus an edge-regular graph. As in Lemma 2.2, we can then deduce that $\Gamma$ is a complete multipartite graph, contrary to our assumption. So, we have $v+\bar{\lambda}-2 \bar{k}>0$.

Lemma 2.19 We have $(\bar{s} v-\bar{k}(\bar{s}+1))(v-\bar{k}-1)=(v-\bar{s}-1)(v+\bar{\lambda}-2 \bar{k}) \bar{s}$.

Proof This is equivalent with $(v+\bar{\lambda}-2 \bar{k}) \bar{s}^{2}+\left(\bar{k}^{2}-\bar{k}+\bar{\lambda}-\bar{\lambda} v\right) \bar{s}-\bar{k}(v-\bar{k}-1)=0$.

Lemma 2.20 We have $v \neq \bar{s}+1$.

Proof If $v=\bar{s}+1$, then Lemmas 2.17 and 2.19 imply that $\bar{k}=\bar{s}$. So, $v=\bar{k}+1$, which is in contradiction with Lemma 2.17.

In view of Lemma 2.20, we can define the following number:

$$
\bar{e}:=\frac{(\bar{s}+1)(\bar{k}-\bar{s})}{v-\bar{s}-1} .
$$


Lemma 2.21 We have $X^{2}+(\bar{\mu}-\bar{\lambda}) X+(\bar{\mu}-\bar{k})=\left(X-\theta_{m}\right)\left(X-\theta_{M}\right)$.

Proof As $\theta_{m} \theta_{M}=\bar{\mu}-\bar{k}$, it suffices to prove that $\theta_{m}=-\frac{\bar{k}}{\bar{s}}$ is a root of $X^{2}+(\bar{\mu}-\bar{\lambda}) X+(\bar{\mu}-\bar{k})$, or equivalently that

$$
\bar{k}^{2}-(\bar{\mu}-\bar{\lambda}) \bar{k} \bar{s}+(\bar{\mu}-\bar{k}) \bar{s}^{2}=0 .
$$

As $\bar{\mu}=\frac{\bar{k}(\bar{k}-\bar{\lambda}-1)}{v-\bar{k}-1}$, we thus need to verify that

$\bar{k}^{2}(v-\bar{k}-1)-(\bar{k}(\bar{k}-\bar{\lambda}-1)-\bar{\lambda}(v-\bar{k}-1)) \bar{k} \bar{s}+(\bar{k}(\bar{k}-\bar{\lambda}-1)-\bar{k}(v-\bar{k}-1)) \bar{s}^{2}=0$.

After division by $-\bar{k}$, the latter equation is equivalent with $(v+\bar{\lambda}-2 \bar{k}) \bar{s}^{2}+\left(\bar{k}^{2}-\bar{k}+\bar{\lambda}-\right.$ $\bar{\lambda} v) \bar{s}-\bar{k}(v-\bar{k}-1)=0$, from which we indeed know that that this is correct.

If $\Gamma$ is a strongly regular graph with parameters $(v, k, \lambda, \mu)$, then we know that the eigenvalues of $\Gamma$ distinct from $k$ are the roots of $X^{2}+(\mu-\lambda) X+(\mu-k) \in \mathbb{R}[X]$, i.e. they are equal to $\theta_{m}$ and $\theta_{M}$.

Lemma 2.22 We have $\theta_{m}<0<\theta_{M}$.

Proof Obviously, $\theta_{m}=-\frac{\bar{k}}{\bar{s}}<0$ and $\theta_{M}=\frac{\bar{k}-\bar{\mu}}{\bar{k}} \bar{s}=\frac{\bar{s}(v+\bar{\lambda}-2 \bar{k})}{v-\bar{k}-1}>0$ by Lemmas 2.17 and 2.18 .

Lemma 2.23 We have $\theta_{M}=\bar{s}-\bar{e}$.

Proof We compute that $\bar{s}-\bar{e}=\bar{s}-\frac{(\bar{s}+1)(\bar{k}-\bar{s})}{v-\bar{s}-1}=\frac{\bar{s} v-\bar{k}(\bar{s}+1)}{v-\bar{s}-1}$. In order for this to be equal to $\theta_{M}=\frac{\bar{k}-\bar{\mu}}{\bar{k}} \bar{s}=\frac{(v+\bar{\lambda}-2 \bar{k}) \bar{s}}{v-\bar{k}-1}$, we must have that $(\bar{s} v-\bar{k}(\bar{s}+1))(v-\bar{k}-1)=(v-\bar{s}-1)(v+$ $\bar{\lambda}-2 \bar{k}) \bar{s}$. By that is precisely Lemma 2.19 .

Lemma 2.24 We have $\bar{k}>\theta_{M}, \bar{k}=\theta_{M}$ or $\bar{k}<\theta_{M}$, depending on whether $\bar{k}>\bar{\lambda}+1$, $\bar{k}=\bar{\lambda}+1$ or $\bar{k}<\bar{\lambda}+1$, or equivalently, depending on whether $\bar{\mu}>0, \bar{\mu}=0$ or $\bar{\mu}<0$.

Proof Taking into account Lemma 2.21 and the definition of $\bar{\mu}$, we see that $\left(\bar{k}-\theta_{m}\right)\left(\bar{k}-\theta_{M}\right)=$ $\bar{k}^{2}+(\bar{\mu}-\bar{\lambda}) \bar{k}+(\bar{\mu}-\bar{k})=\bar{\mu} v$. As $\theta_{m}<0$, we have $\bar{k}-\theta_{m}>0$ and so $\bar{k}-\theta_{M}$ and $\bar{\mu}=\frac{\bar{k}(\bar{k}-\bar{\lambda}-1)}{v-\bar{k}-1}$ are either both 0 , both negative or both positive.

The following lemma will be useful in our discussion. It is precisely Theorem 3.2.1 of [4] applied to the graph $\Gamma$.

Lemma 2.25 The largest eigenvalue of $\Gamma$ is at least $\bar{k}$ with equality if and only if $\Gamma$ is regular.

Lemma 2.26 If $\Gamma$ is regular, then $\bar{k} \geq \bar{\lambda}+1$.

Proof For a regular graph $\Gamma$ of degree $k:=\bar{k}$, we have $\lambda_{x y}+1=\left|\{y\} \cup\left(\Gamma_{1}(x) \cap \Gamma_{1}(y)\right)\right| \leq$ $\left|\Gamma_{1}(x)\right|=k$ for every edge $x y$ of $\Gamma$, implying that $\bar{\lambda}+1 \leq k$.

If $\Gamma$ is a strongly regular graph, we thus know that $\bar{k} \geq \bar{\lambda}+1$ and that $\theta_{m}$ is the smallest eigenvalue. In fact, we can prove the following.

Lemma 2.27 Suppose $\bar{k} \geq \bar{\lambda}+1$ and let $\theta_{\text {min }}$ denote the smallest eigenvalue of $\Gamma$. Then $\theta_{\min } \leq \theta_{m}$ and equality holds if and only if $\Gamma$ is a strongly regular graph. 
Proof Let $\theta^{*}$ be the largest eigenvalue of $\Gamma$, and put $\Omega^{\prime}:=\Omega_{\theta^{*}}$. By Lemmas 2.24 and 2.25, we have $\theta^{*} \geq \bar{k} \geq \theta_{M}$. By relying on Lemmas 2.16 and 2.21 , we compute

$$
\begin{aligned}
& \sum_{\omega \in \Omega}\left(\omega-\theta_{M}\right)^{2}\left(\omega-\theta_{m}\right) \\
& =\sum_{\omega \in \Omega}\left(\omega^{2}+(\bar{\mu}-\bar{\lambda}) \omega+(\bar{\mu}-\bar{k})\right)\left(\omega-\theta_{M}\right) \\
& =\sum_{\omega \in \Omega}\left(\omega^{3}+\left(\bar{\mu}-\bar{\lambda}-\theta_{M}\right) \omega^{2}+\left(\bar{\mu}-\bar{k}-\theta_{M}(\bar{\mu}-\bar{\lambda})\right) \omega-\theta_{M}(\bar{\mu}-\bar{k})\right) \\
& =v \bar{k} \bar{\lambda}+\left(\bar{\mu}-\bar{\lambda}-\frac{\bar{k}-\bar{\mu}}{\bar{k}} \bar{s}\right) v \bar{k}-\frac{\bar{k}-\bar{\mu}}{\bar{k}} \bar{s}(\bar{\mu}-\bar{k}) v \\
& =\bar{\mu} v \bar{k}-\frac{\bar{\mu}(\bar{k}-\bar{\mu}) \bar{s} v}{\bar{k}} .
\end{aligned}
$$

This number is equal to

$$
\left(\bar{k}-\theta_{M}\right)^{2}\left(\bar{k}-\theta_{m}\right)=\left(\bar{k}^{2}+(\bar{\mu}-\bar{\lambda}) \bar{k}+(\bar{\mu}-\bar{k})\right)\left(\bar{k}-\theta_{M}\right)=\bar{\mu} v\left(\bar{k}-\theta_{M}\right)=\bar{\mu} v\left(\bar{k}-\frac{\bar{k}-\bar{\mu}}{\bar{k}} \bar{s}\right) .
$$

As the map $\mathbb{R} \rightarrow \mathbb{R} ; x \mapsto\left(x-\theta_{M}\right)^{2}\left(x-\theta_{m}\right)$ attains a local maximum for $x=\frac{2 \theta_{m}+\theta_{M}}{3}<$ $\theta_{M}$ and a local minimum for $x=\theta_{M} \leq \bar{k} \leq \theta^{*}$, we have that

$$
\left(\theta^{*}-\theta_{M}\right)^{2}\left(\theta^{*}-\theta_{m}\right) \geq\left(\bar{k}-\theta_{M}\right)^{2}\left(\bar{k}-\theta_{m}\right)=\sum_{\omega \in \Omega}\left(\omega-\theta_{M}\right)^{2}\left(\omega-\theta_{m}\right),
$$

i.e.

$$
\sum_{\omega \in \Omega^{\prime}}\left(\omega-\theta_{M}\right)^{2}\left(\omega-\theta_{m}\right) \leq 0 .
$$

By Lemma 2.15(a)+(b) and the fact that $\Gamma$ is not complete nor a graph without edges, we know that the multiset $\Omega^{\prime}=\Omega_{\theta^{*}}$ contains at least two distinct elements. Equation (10) then implies that there exists an $\omega \in \Omega^{\prime}$ with $\omega \leq \theta_{m}$, i.e. $\theta_{\text {min }} \leq \theta_{m}$.

Suppose now that $\theta_{\min }=\theta_{m}$. Then $\omega \geq \theta_{m}$ for every $\omega \in \Omega^{\prime}$ and so $\sum_{\omega \in \Omega^{\prime}}(\omega-$ $\left.\theta_{M}\right)^{2}\left(\omega-\theta_{m}\right) \geq 0$. In combination with (10), this implies that $\omega \in\left\{\theta_{M}, \theta_{m}\right\}$ for every $\omega \in \Omega^{\prime}$. If $\theta^{*} \neq \overline{\bar{k}}$, then the inequalities in (9) and (10) would be strict, which is impossible. So, $\theta^{*}=\bar{k}$, and every eigenvalue of $\Gamma$ is equal to $\bar{k}, \theta_{m}$ or $\theta_{M}$. The fact that $\theta^{*}=\bar{k}$ implies by Lemma 2.25 that $\Gamma$ is regular with valency $k:=\bar{k}$.

If $\Gamma$ is connected, then $\Gamma$ is strongly regular by Lemma 2.15 . If $\Gamma$ is not connected, then each connected component of $\Gamma$ is regular of degree $k$ and as each $\omega \in \Omega_{\theta^{*}}=\Omega_{k}$ belongs to $\left\{\theta_{M}, \theta_{m}\right\}$ we then know that $\Gamma$ has at most two eigenvalues, implying by Lemma $2.15(\mathrm{a})+(\mathrm{b})$ that $\Gamma$ is a disjoint union of at least two complete graphs of the same order. In this case, $\Gamma$ is thus also a strongly regular graph.

The following is now a consequence of Lemmas 2.26 and 2.27.

Corollary 2.28 If $\Gamma$ is regular with smallest eigenvalue $\theta_{\min }$, then $\theta_{\min } \leq \theta_{m}$ with equality if and only if $\Gamma$ is strongly regular.

If $\Gamma$ is a strongly regular graph with valency $k$, then we know that $\theta_{M}$ is the largest eigenvalue in $\Omega_{k}$. In fact, the following can be proved. 
Lemma 2.29 Suppose $\Gamma$ is regular with valency $k:=\bar{k}$ and let $\theta_{\text {Max }}$ denote the largest eigenvalue of $\Gamma$ in $\Omega^{\prime}:=\Omega_{k}$. Then $\theta_{\text {Max }} \geq \theta_{M}$ with equality if and only if $\Gamma$ is strongly regular.

Proof By relying on Lemmas 2.16 and 2.21, we compute

$$
\begin{aligned}
& \sum_{\omega \in \Omega}\left(\omega-\theta_{M}\right)\left(\omega-\theta_{m}\right)^{2} \\
& =\sum_{\omega \in \Omega}\left(\omega^{2}+(\bar{\mu}-\bar{\lambda}) \omega+(\bar{\mu}-\bar{k})\right)\left(\omega-\theta_{m}\right) \\
& =\sum_{\omega \in \Omega}\left(\omega^{3}+\left(\bar{\mu}-\bar{\lambda}-\theta_{m}\right) \omega^{2}+\left(\bar{\mu}-\bar{k}-\theta_{m}(\bar{\mu}-\bar{\lambda})\right) \omega-\theta_{m}(\bar{\mu}-\bar{k})\right) \\
& =v \bar{k} \bar{\lambda}+\left(\bar{\mu}-\bar{\lambda}+\frac{\bar{k}}{\bar{s}}\right) v \bar{k}+\frac{\bar{k}}{\bar{s}}(\bar{\mu}-\bar{k}) v \\
& =\bar{\mu} v \bar{k}+\frac{\bar{\mu} v \bar{k}}{\bar{s}} .
\end{aligned}
$$

This number is equal to

$$
\left(\bar{k}-\theta_{M}\right)\left(\bar{k}-\theta_{m}\right)^{2}=\left(\bar{k}^{2}+(\bar{\mu}-\bar{\lambda}) \bar{k}+(\bar{\mu}-\bar{k})\right)\left(\bar{k}-\theta_{m}\right)=\bar{\mu} v\left(\bar{k}-\theta_{m}\right)=\bar{\mu} v\left(\bar{k}+\frac{\bar{k}}{\bar{s}}\right) .
$$

We thus have

$$
\sum_{\omega \in \Omega^{\prime}}\left(\omega-\theta_{M}\right)\left(\omega-\theta_{m}\right)^{2}=0 .
$$

By Lemma 2.15(a) + (b) and the fact that $\Gamma$ is not complete nor a graph without edges, we know that the multiset $\Omega^{\prime}=\Omega_{k}$ contains at least two distinct elements. Equation (11) then implies that there exists an $\omega \in \Omega^{\prime}$ with $\omega \geq \theta_{M}$, i.e. $\theta_{M a x} \geq \theta_{M}$.

Suppose now that $\theta_{\operatorname{Max}}=\theta_{M}$. Then $\omega \leq \theta_{M}$ for every $\omega \in \Omega^{\prime}$. In combination with (11), this implies that $\omega \in\left\{\theta_{M}, \theta_{m}\right\}$ for every $\omega \in \Omega^{\prime}$.

If $\Gamma$ is connected, then $\Gamma$ is strongly regular by Lemma 2.15. If $\Gamma$ is not connected, then each connected component of $\Gamma$ is regular of degree $k$ and as each $\omega \in \Omega^{\prime}=\Omega_{k}$ belongs to $\left\{\theta_{M}, \theta_{m}\right\}$, we then know that $\Gamma$ has at most two eigenvalues, implying by Lemma $2.15(\mathrm{a})+$ (b) that $\Gamma$ is a disjoint union of at least two complete graphs of the same order. In this case, $\Gamma$ is thus also a strongly regular graph.

Theorem 2.14 is now implied by Corollary 2.28 and Lemma 2.29.

\subsection{Characterizations using the Hoffman's ratio bound}

Delsarte [7, p. 31] showed that if $C$ is a clique in a strongly regular graph $\Gamma$ with parameters $(v, k, \lambda, \mu)$, then

$$
|C| \leq 1-\frac{k}{\theta_{\min }}
$$

with $\theta_{\min }$ the smallest eigenvalue of $\Gamma$. A coclique $C^{\prime}$ in $\Gamma$ can be regarded as a clique in the complementary graph $\bar{\Gamma}$ of $\Gamma$ and so we have

$$
\left|C^{\prime}\right| \leq 1-\frac{v-k-1}{\theta_{C \min }}=\frac{v}{1-\frac{k}{\theta_{\min }}},
$$


with $\theta_{\text {Cmin }}$ the smallest eigenvalue of $\bar{\Gamma}$. We call a (co)clique that meets the Delsarte bound in (12) or (13) a Delsarte-(co)clique. Note that many people call them Hoffman-(co)cliques. The bound for strongly regular graphs, however, was first given by Delsarte. Hoffman later generalized it to arbitrary regular graphs, and the bound is often called the ratio bound. Specifically, Hoffman showed that

$$
\left|C^{\prime}\right| \leq v\left(1-\frac{k}{\theta_{\min }}\right)^{-1}
$$

for any coclique $C^{\prime}$ in a regular graph with valency $k \geq 1$ on $v$ vertices having smallest eigenvalue $\theta_{\min }$, with equality if and only if every vertex outside $C$ has the same number of neighbours in $C$. A similar characterization for the equality case holds for the inequality (12). For proofs and more background information on these bounds, see [1, Propositions 1.3.2 and 4.4.6].

Theorem 2.30 Let $\Gamma=(V, E)$ be a connected, non-complete edge-regular graph. Then the following statements are equivalent:

(1) $\Gamma$ is a strongly regular Neumaier graph;

(2) there exists a clique $C$ in $\Gamma$ such that $|C|$ attains the Hoffman coclique bound in the complementary graph $\bar{\Gamma}$.

Proof Let $v, k$ and $\lambda$ be the parameters of $\Gamma$ as an edge-regular graph, and define $s$ as in the beginning of Sect. 2.1.

$(2) \Rightarrow(1)$. In case $\Gamma$ is a complete multipartite graph, it must be a strongly regular Neumaier graph, see e.g. Sect. 2.1. So, for this part of the proof, we may assume that $\Gamma$ is not a complete multipartite graph. Suppose there exists a clique $C$ in $\Gamma$ such that $|C|$ attains the Hoffman coclique bound in $\bar{\Gamma}$. Then

$$
|C|=\frac{v}{1+\frac{v-k-1}{-\theta_{\text {Cmin }}}}=\frac{v}{1+\frac{v-k-1}{\theta_{\operatorname{Max} 2}+1}},
$$

where $\theta_{C \min }$ is the smallest eigenvalue of $\bar{\Gamma}$. Note that $-\theta_{\text {Cmin }}=\theta_{\operatorname{Max} 2}+1$, where $\theta_{\operatorname{Max} 2}$ is the second largest eigenvalue of $\Gamma$, see e.g. Theorem 2.6 of [3]. We also know that every vertex of $V \backslash C$ is $\bar{\Gamma}$-adjacent to a constant number of vertices of $C$, or equivalently, $\Gamma$ adjacent to a constant number $e$ of vertices of $C$. As $\Gamma$ is connected and non-complete, $e>0$ and so $C$ is a regular clique. By Proposition 2.3, we then know that $|C|=s+1$ and $e=\frac{(s+1)(k-s)}{v-(s+1)}$. The quotient matrix $\left[\begin{array}{ll}s & k-s \\ e & k-e\end{array}\right]$ of the equitable partition $\{C, V \backslash C\}$ has then $k$ and $s-e$ as eigenvalues. So, $k$ and $s-e$ are also eigenvalues of $\Gamma$ and $\theta_{\operatorname{Max} 2} \geq s-e$. As $s-e+1=\frac{(s+1)(v-(k+1))}{v-(s+1)}$, we have

$s+1=|C|=\frac{v}{1+\frac{v-k-1}{\theta_{\operatorname{Max} 2}+1}} \geq \frac{v}{1+\frac{v-k-1}{s-e+1}}=\frac{v}{1+\frac{v-(s+1)}{s+1}}=\frac{v(s+1)}{s+1+v-(s+1)}=s+1$,

implying that $\theta_{\operatorname{Max} 2}=s-e$. So, the second largest eigenvalue of $\Gamma$ is $s-e$, implying by Theorem 2.14(2) and Lemma 2.23 that $\Gamma$ is strongly regular. As $\Gamma$ has a regular clique, it is a Neumaier graph.

$(1) \Rightarrow(2)$. Suppose $\Gamma$ is a strongly regular Neumaier graph. If $\Gamma$ is not a complete multipartite graph, then by Lemmas 2.21 and 2.23, the eigenvalues of $\Gamma$ are $k, s-e$ and $-\frac{k}{s}$. In fact, by direct verification one can see that this remains true for complete multipartite 
graphs. So, $\theta_{\operatorname{Max} 2}=s-e$. The upper bound in the Hoffman coclique bound is then equal to

$$
\frac{v}{1+\frac{v-k-1}{\theta_{\text {Max } 2}+1}}=\frac{v}{1+\frac{v-k-1}{s-e+1}}=\frac{v}{1+\frac{v-(s+1)}{s+1}}=\frac{v(s+1)}{s+1+v-(s+1)}=s+1 .
$$

Every regular clique in $\Gamma$ has order $s+1$, and so there exists a coclique in $\bar{\Gamma}$ attaining the Hoffman coclique bound.

Theorem 2.31 The following are equivalent for a Neumaier graph $\Gamma$.

(1) $\Gamma$ is strongly regular.

(2) The Delsarte clique bound holds for $\Gamma$, i.e. $|C| \leq 1-\frac{k}{\theta_{\min }}$ for every clique $C$, with $k$ the valency of $\Gamma$ and $\theta_{\min }$ the smallest eigenvalue of $\Gamma$.

Proof Let $v, k$ and $\lambda$ be the parameters of $\Gamma$ as an edge-regular graph, and define $s$ and $e$ as in the beginning of Sect. 2.1. If $C$ is a clique of $\Gamma$, then by Propositions 2.3 and 2.4 we know that $|C| \leq s+1$, with equality if and only if $C$ is a regular clique.

$(1) \Rightarrow(2)$. Suppose $\Gamma$ is strongly regular. Similarly as in Theorem 2.30, we then know that $k, s-e$ and $-\frac{k}{s}$ are the eigenvalues of $\Gamma$. So, $1-\frac{k}{\theta_{\min }}=s+1$. As $|C| \leq s+1$ for every clique $C$, we indeed see that the Delsarte clique bound holds in $\Gamma$.

$(2) \Rightarrow(1)$. Suppose the Delsarte clique bound holds in $\Gamma$. If $C$ is a regular clique in $\Gamma$, then $s+1=|C| \leq 1-\frac{k}{\theta_{\min }}$, implying that $\theta_{\min } \geq-\frac{k}{s}$. Then by Theorem 2.14 (a) Corollary 2.28 it follows that $\Gamma$ is strongly regular.

\subsection{A characterization using $t$-walk regularity}

Recall that a $t$-walk-regular graph is a graph $\Gamma$ for which the number of walks of a given length between two vertices depends only on the distance between these two vertices, as long as this distance is at most $t$. Such graphs generalize distance-regular graphs and $t$-arc-transitive graphs. Every $t$-walk-regular graph is also regular.

Let $\Gamma$ be a connected regular graph with distinct eigenvalues $k=\theta_{0}, \theta_{1}, \ldots, \theta_{d}$ and adjacency matrix $A$. Let $R_{i}:=\frac{\prod_{j: j \neq i}\left(A-\theta_{j} I\right)}{\prod_{j: j \neq i} \theta_{i}-\theta_{j}}$ with $i \in\{0,1, \ldots, d\}$ be the primitive idempotent of $\Gamma$ corresponding to the eigenvalue $\theta_{i}$. We have $A R_{i}=\theta_{i} R_{i}$. Let $A_{i}$ be the $i$-adjacency matrix, i.e. $\left(A_{i}\right)_{x y}=1$ if $d(x, y)=i$ and 0 otherwise.

By Theorem 3.1 of [5], we know that $\Gamma$ is $t$-walk-regular if and only if for every $i \in$ $\{0,1, \ldots, t\}$ and every $j \in\{0,1, \ldots, d\}$, there exists an $\alpha_{i j} \in \mathbb{R}$ such that $A_{i} \circ R_{j}=\alpha_{i j} A_{i}$, where $\circ$ denotes the entrywise product. From now on we assume that $\Gamma$ is 1-walk-regular. For every $i \in\{0,1, \ldots, d\}$, we define $\epsilon_{i}:=\left(R_{i}\right)_{x x}$ where $x$ is any vertex of $\Gamma$, and put $\tilde{R}_{i}:=\frac{1}{\epsilon_{i}} R_{i}$. It is easy to see that $\left(\tilde{R}_{i}\right)_{x y}=\theta_{i} / k$ for adjacent vertices $x$ and $y$.

Neumaier [13, Corollary 2.4] showed that a vertex- and edge-transitive graph that has a regular clique must be strongly regular. Since vertex- and edge-regularity implies 1-walkregularity, the next theorem provides an analogous of Neumaier's result, but it requires a weaker assumption. For more properties of 1-walk-regular graphs we refer the reader to [2].

Theorem 2.32 Let $\Gamma=(V, E)$ be a 1-walk-regular graph with a regular clique. Then $\Gamma$ is a strongly regular graph.

Proof Let $k$ be the valency of $\Gamma$. Let $C$ be a regular clique of size $s+1$ and suppose every vertex outside $C$ has $e$ neighbours in $C$. As $e \geq 1$, the graph $\Gamma$ is connected. Consider the

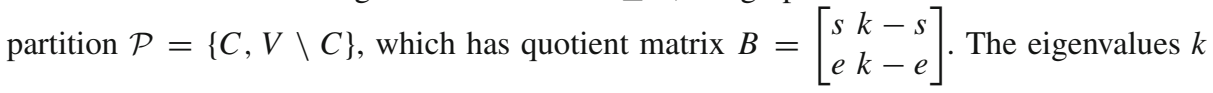


and $s-e<k$ of $B$ are also eigenvalues of the adjacency matrix $A$ of $\Gamma$. If [ $\left.v_{1} v_{2}\right]^{T}$ is an eigenvector of $B$ corresponding to $s-e$, then $v_{1} \neq v_{2}$ and the column matrix $\omega$ of size $|V|$ with $\omega_{x}=v_{1}$ if $x \in C$ and $\omega_{x}=v_{2}$ otherwise is an eigenvector of $A$ corresponding to the eigenvector $s-e$. Note that $j=\left[\begin{array}{llll}1 & 1 & \cdots & 1\end{array}\right]^{T}$ is an eigenvector of $A$ corresponding to $k$. The characteristic vector $\chi_{C}$ of $C$ is a linear combination of $\omega$ and $j$.

Assume now that $\theta_{i} \notin\{k, s-e\}$ is another eigenvalue, with idempotent $R_{i}$, and consider the matrix $\tilde{R}_{i}$ as defined above. Any column of $\tilde{R}_{i}$ is orthogonal to $\omega$ and $j$ and hence also to $\chi_{C}$. Considering a column of $\tilde{R}_{i}$ corresponding to a vertex of $C$, we then find that $1+s u=0$, where $u=\frac{\theta_{i}}{k}$. The eigenvalue $\theta_{i}$ of $\Gamma$ is thus uniquely determined. It follows that $\Gamma$ has at most three distinct eigenvalues. As $\Gamma$ is also connected and regular, it must be a strongly regular graph.

Theorem 2.32 has the following immediate corollary.

Corollary 2.33 It is not possible to construct a Neumaier graph as a relation graph of a symmetric association scheme that does not come from a strongly regular graph.

It is worth noting that there are vertex-transitive Neumaier graphs that are not strongly regular graphs, see for instance the construction provided by Greaves and Koolen [11] which uses Cayley graphs. Note that vertex-transitivity implies 0-walk-regular.

It is also important to note that a vertex-transitive Neumaier graph has diameter 2, and it is not known whether this is still true under the weaker condition of walk-regularity. Examples of Neumaier graphs with diameter 3 are known [9].

\subsection{A characterization using the smallest eigenvalue being -2}

In [1, Theorem 3.12.4] it is shown that if a connected graph is edge-regular with smallest eigenvalue -2 , then it is strongly regular or the line graph of a triangle-free regular graph.

Proposition 2.34 Suppose the line graph $L(\Gamma)$ of a graph $\Gamma$ is a Neumaier graph. Then $L(\Gamma)$ is one of the following:

(1) an $(s+1) \times(s+1)$ rook graph for some $s \in \mathbb{N} \backslash\{0\}$;

(2) a Johnson graph $J(s+2,2)$ for some $s \in \mathbb{N} \backslash\{0,1\}$;

(3) the octahedral graph.

In particular, $L(\Gamma)$ is a strongly regular graph.

Proof As adding isolated vertices to a graph does not alter its line graph, we may without loss of generality assume that $\Gamma$ does not contain isolated vertices. As $L(\Gamma)$ is connected, the graph $\Gamma$ must therefore be connected as well. We denote by $k$ the valency of $L(\Gamma)$ and by $\lambda$ the constant number of triangles through a given edge of $L(\Gamma)$. We also assume that $C$ is a regular clique of size $s+1$ of $L(\Gamma)$ and that every vertex of $L(\Gamma)$ not in $C$ is adjacent to exactly $e>0$ vertices of $C$. As $C$ is a maximal clique, it consists either of all the edges in $\Gamma$ containing a given vertex or of all three edges in a triangle of $\Gamma$.

Suppose first that $C$ consists of all the edges in $\Gamma$ through a vertex $v$. Every edge of $\Gamma$ not incident with $v$ is adjacent to at most two edges in $C$, implying that $e \in\{1,2\}$.

Assume $e=1$. Then $\Gamma(v)$ cannot contain edges. If $v^{\prime}$ is a vertex not belonging to $\{v\} \cup \Gamma(v)$, then the condition $e=1$ implies that every edge through $v^{\prime}$ is of the form $v^{\prime} w$ with $w$ one of the $s+1$ vertices of $\Gamma(v)$. The degree $k$ of the edges $v w$ and $v^{\prime} w$ in $L(\Gamma)$ is equal to $\operatorname{deg}(v)+\operatorname{deg}(w)-2=\operatorname{deg}\left(v^{\prime}\right)+\operatorname{deg}(w)-2$, implying that $\operatorname{deg}\left(v^{\prime}\right)=\operatorname{deg}(v)=s+1$. 
This implies that $\Gamma \cong K_{s+1, n}$ for some $n \in \mathbb{N} \backslash\{0,1\}$. Edge-regularity in $L(\Gamma)$ implies that $n=s+1$ and that $L(\Gamma)$ is the $(s+1) \times(s+1)$ rook graph.

Assume $e=2$. Then all edges not incident with $v$ are contained in $\Gamma(v)$, and so $\{v\} \cup \Gamma(v)$ is the whole vertex set of $\Gamma$. If $b=w_{1} w_{2}$ is an edge not incident with $v$, then the edge-regularity condition applied to respectively $\left\{v w_{1}, v w_{2}\right\}$ and $\left\{v w_{1}, w_{1} w_{2}\right\}$ gives $\lambda=\operatorname{deg}(v)-2+1=$ $\operatorname{deg}\left(w_{1}\right)-2+1$, implying that $\operatorname{deg}\left(w_{1}\right)=\operatorname{deg}(v)=s+1$. Repeating this argument for other edges not incident with $v$, we see that all vertices of $\Gamma$ have degree $s+1$. So, $\Gamma \cong K_{s+2}$ and $L(\Gamma) \cong J(s+2,2)$. As $L(\Gamma)$ is not complete, we have $s \geq 2$.

Suppose next that $C=\left\{v_{1} v_{2}, v_{1} v_{3}, v_{2} v_{3}\right\}$ where $\Delta=\left\{v_{1}, v_{2}, v_{3}\right\}$ is a triangle of $\Gamma$. The valency $k$ of $L(\Gamma)$ is equal to $\operatorname{deg}\left(v_{1}\right)+\operatorname{deg}\left(v_{2}\right)-2=\operatorname{deg}\left(v_{1}\right)+\operatorname{deg}\left(v_{3}\right)-2=$ $\operatorname{deg}\left(v_{2}\right)+\operatorname{deg}\left(v_{3}\right)-2$, implying that $\operatorname{deg}\left(v_{1}\right), \operatorname{deg}\left(v_{2}\right)$ and $\operatorname{deg}\left(v_{3}\right)$ are equal, say to $l$, and that $k=2 l-2$. Since $\Gamma$ is connected and has edges outside $C$, we have $l \geq 3$. Note that the set of $l \geq 3$ edges through $v_{1}$ is a clique of $L(\Gamma)$. As the maximal size of a clique of $L(\Gamma)$ is equal to $|C|=3$, we have $l=3$ and $k=4$. Every vertex $v_{i} \in\left\{v_{1}, v_{2}, v_{3}\right\}$ is therefore incident with a unique edge $e_{i}$ not contained in $\Delta$. We see that $e_{i}$ and hence all vertices $b \notin C$ are adjacent with precisely $e=2$ edges of $C$. So, $e_{1}, e_{2}$ and $e_{3}$ are the only edges not in $C$. As the degree of $e_{1}$ in $L(\Gamma)$ equals $k=4$, the edges $e_{1}, e_{2}$ and $e_{3}$ are mutually adjacent, implying that $\Gamma$ is the tetrahedral graph and $L(\Gamma)$ is the octahedral graph.

Proposition 2.34 and [[1], Theorem 3.12.4] imply the following straightforward consequence.

Corollary 2.35 Every Neumaier graph with smallest eigenvalue -2 is strongly regular.

\section{Neumaier graphs with four eigenvalues}

Regular graphs with precisely four eigenvalues have already been studied in the literature, see e.g. [14]. In this section, we prove that no such graph can be a Neumaier graph.

\section{Theorem 3.1 There are no Neumaier graphs with exactly four distinct eigenvalues.}

Proof Suppose to the contrary that $\Gamma$ is a Neumaier graph with exactly four eigenvalues $k$, $\theta_{1}, \theta$ and $\theta_{2}$, with $k$ the valency of $\Gamma$ and $k>\theta_{1}>\theta>\theta_{2}$. Suppose $C$ is a regular clique of size $s+1$ and every vertex outside $C$ is adjacent to precisely $e>0$ vertices of $C$. Above, we have already seen that $e=\frac{(s+1)(k-s)}{v-s-1}$ with $v$ the number of vertices of $\Gamma$, i.e.

$$
v=\frac{(s+1)(k-s+e)}{e} .
$$

Counting triangles having exactly one of its edges in $C$, we find

$$
\left(\begin{array}{c}
s+1 \\
2
\end{array}\right)(\lambda-(s-1))=(v-s-1)\left(\begin{array}{l}
e \\
2
\end{array}\right),
$$

with $\lambda$ denoting the constant number of triangles containing a given edge.

The equitable partition $\{C, V \backslash C\}$ has quotient matrix $\left[\begin{array}{ll}s & k-s \\ e & k-e\end{array}\right]$, whose eigenvalues are $k$ and $s-e$. As these are also eigenvalues of $\Gamma$, we have $s-e \in\left\{\theta, \theta_{1}, \theta_{2}\right\}$. In fact, we can prove that $s-e=\theta$. As $C$ cannot be properly contained in another clique, we have $e \leq s$. So, $s-e \geq 0$ is distinct from the smallest eigenvalue $\theta_{2}$ of $\Gamma$ which is negative. As $\Gamma$ has 
exactly four eigenvalues, it is not a strongly regular graph, implying by Theorem 2.14 and Lemma 2.23 that $\theta_{1}>s-e$. Summarizing we thus have

$$
k>\theta_{1}>\theta=s-e \geq 0>\theta_{2} .
$$

As $s=\theta+e$, equations (15) and (16) become

$$
v=\frac{(\theta+e+1)(k-\theta)}{e}, \quad \lambda=\theta+e-1+\frac{(k-\theta-e)(e-1)}{\theta+e} .
$$

As $|C|=s+1=\theta+e+1$ and $\Gamma$ is not strongly regular, Theorem 2.31 implies that $\theta_{2}<-\frac{k}{\theta+e}$, or equivalently

$$
k+e \theta_{2}+\theta_{2} \theta<0 .
$$

The eigenvalues of the adjacency matrix $A$ of $\Gamma$ distinct from $k$ are $\theta, \theta_{1}$ and $\theta_{2}$. As $\Gamma$ is connected and regular with valency $k$, we thus have

$$
(A-\theta I)\left(A-\theta_{1} I\right)\left(A-\theta_{2} I\right)=\alpha J
$$

for some $\alpha \in \mathbb{R}$, where $J$ denotes the $v \times v$ matrix with all entries equal to 1 . Equation (17) can be rewritten as

$$
A^{3}+\beta_{2} A^{2}+\beta_{1} A+\beta_{0} I=\alpha J
$$

where $\beta_{2}=-\left(\theta+\theta_{1}+\theta_{2}\right), \beta_{1}=\theta \theta_{1}+\theta \theta_{2}+\theta_{1} \theta_{2}$ and $\beta_{0}=-\theta \theta_{1} \theta_{2}$. If we calculate the diagonal entries of the matrices occurring at both sides of (18), we find that

$$
\alpha=k \lambda+\beta_{2} k+\beta_{0}=k \lambda+\left(-\theta-\theta_{1}-\theta_{2}\right) k-\theta \theta_{1} \theta_{2},
$$

as there are $k$ and $k \lambda$ closed walks of respective lengths 2 and 3 starting and ending in a given vertex of $\Gamma$. On the other hand, after multiplying both sides of (17) by $j=\left[\begin{array}{llll}1 & 1 & \cdots\end{array}\right]^{T}$, we find

$$
(k-\theta)\left(k-\theta_{1}\right)\left(k-\theta_{2}\right)=\alpha v=\left(k \lambda+\left(-\theta-\theta_{1}-\theta_{2}\right) k-\theta \theta_{1} \theta_{2}\right) \frac{(\theta+e+1)(k-\theta)}{e},
$$
i.e.

$$
\left(k-\theta_{1}\right)\left(k-\theta_{2}\right)=\left(k \lambda+\left(-\theta-\theta_{1}-\theta_{2}\right) k-\theta \theta_{1} \theta_{2}\right) \frac{(\theta+e+1)}{e} .
$$

The latter equation implies that

$$
\left(\theta_{2} e+k \theta+k+\theta_{2} \theta^{2}+e \theta_{2} \theta+\theta_{2} \theta\right) \theta_{1}=-k^{2} e+k \theta_{2} e+k(\theta+e+1)\left(\lambda-\theta-\theta_{2}\right) .
$$

As $\theta_{2} e+k \theta+k+\theta_{2} \theta^{2}+e \theta_{2} \theta+\theta_{2} \theta=(\theta+1)\left(k+e \theta_{2}+\theta_{2} \theta\right) \neq 0$, we thus obtain

$$
\begin{aligned}
\theta_{1} & =\frac{-k^{2} e+k \theta_{2} e+k(\theta+e+1)\left(e-1+\frac{(k-\theta-e)(e-1)}{\theta+e}-\theta_{2}\right)}{(\theta+1)\left(k+e \theta_{2}+\theta_{2} \theta\right)} \\
& =\frac{-k\left(k \theta+k+\theta_{2} \theta+\theta_{2} \theta^{2}+e \theta_{2}+e \theta_{2} \theta\right)}{(\theta+1)\left(k+e \theta_{2}+\theta_{2} \theta\right)(e+\theta)} \\
& =\frac{-k}{e+\theta} .
\end{aligned}
$$

This is impossible as $\theta_{1}>0>-\frac{k}{e+\theta}$. 
Acknowledgements We would like to thank Edwin van Dam for providing insightful remarks that improved the paper. A. Abiad is partially supported by the FWO (Research Foundation Flanders) Grant 1285921N. The research of J. D'haeseleer is supported by the FWO. J.H. Koolen is partially supported by the National Natural Science Foundation of China (No. 12071454), Anhui Initiative in Quantum Information Technologies (No. AHY150000) and the project 'Analysis and Geometry on Bundles' of Ministry of Science and Technology of the People's Republic of China.

Open Access This article is licensed under a Creative Commons Attribution 4.0 International License, which permits use, sharing, adaptation, distribution and reproduction in any medium or format, as long as you give appropriate credit to the original author(s) and the source, provide a link to the Creative Commons licence, and indicate if changes were made. The images or other third party material in this article are included in the article's Creative Commons licence, unless indicated otherwise in a credit line to the material. If material is not included in the article's Creative Commons licence and your intended use is not permitted by statutory regulation or exceeds the permitted use, you will need to obtain permission directly from the copyright holder. To view a copy of this licence, visit http://creativecommons.org/licenses/by/4.0/.

\section{References}

1. Brouwer A.E., Cohen A.M., Neumaier A.: Distance-regular graphs. Springer-Verlag, Berlin (1989).

2. Cámara M., van Dam E.R., Koolen J.H., Park Y.: Geometric aspects of 2-walk-regular graphs. Linear Algebra and its Applications 439(9), 2692-2710 (2013).

3. Cvetković D.M., Doob M., Sachs H.: Spectra of graphs. Theory and applications. Third edition. Johann Ambrosius Barth Verlag, Heidelberg (1995).

4. Cvetković, D., Rowlinson, P., Simić, S.: An introduction to the theory of graph spectra. London Mathematical Society Student Texts 75. Cambridge University Press, Cambridge, (2010).

5. Dalfó C., Fiol M.A., Garriga E.: On $k$-walk-regular graphs. Electron. J. Combin. 16(1), R47 (2009).

6. De Bruyn B., Suzuki H.: Intriguing sets of vertices of regular graphs. Graphs Combin. 26(5), 629-646 (2010).

7. Delsarte, P.: An algebraic approach to the association schemes of coding theory. Philips Res. Rep. Suppl. 10, (1973)

8. Evans R.J., Goryainov S., Panasenko D.: The smallest strictly Neumaier graph and its generalisations. Electron. J. Combin. 26(2), 2.29 (2019).

9. Goryainov, S.: private communication.

10. Goryainov S., Shalaginov L.: Cayley-Deza graphs with fewer than 60 vertices. Sib. Electron. Math. Rep. 11, 268-310 (2014). (in Russian).

11. Greaves G.R.W., Koolen J.H.: Edge-regular graphs with regular cliques. European J. Combin. 71, 194-201 (2018).

12. Greaves G.R.W., Koolen J.H.: Another construction of edge-regular graphs with regular cliques. Discrete Math. 342(10), 2818-2820 (2019).

13. Neumaier, A.: Regular cliques in graphs and special $\frac{1}{2}$-designs. In "Finite geometries and designs, Proc. 2nd Isle of Thorns Conf. 1980, volume 49 of London Math. Soc. Lecture Note Ser., pages 244-259. Cambridge University Press, (1981)

14. van Dam, E.R.: Regular graphs with four eigenvalues. Linear Algebra Appl. 226-228, 139-162 (1995)

15. van Dam E.R., Haemers W.H.: A Characterization of Distance-Regular Graphs with Diameter Three. Journal of Algebraic Combinatorics 6, 299-303 (1997).

Publisher's Note Springer Nature remains neutral with regard to jurisdictional claims in published maps and institutional affiliations. 